\title{
Forgetting Memristor Based Neuromorphic System for Pattern Training and Recognition
}

\author{
Peijian Zhang, Chuandong Li*, Senior Member, IEEE, Tingwen Huang, Senior Member, IEEE, Ling Chen and \\ Yiran Chen
}

\begin{abstract}
This paper presents a neuromorphic system for mean variance based pattern training and recognition. The system contains a self-learning circuit, a training circuit and a recognition circuit. Memristor model with forgetting effect which has memory ability and forgetting effect simultaneously is applied to simulate forgetting mechanism of neuromorphic system. Different from previous work, which divided training circuit as off line process, here the weight-changing circuit and the recognition part are combined on line for pattern training and recognition. For illustration, the whole neuromorphic system is applied to recognize handwriting number ' $0-9$ ' on gray images, and simulations verify its effectiveness.
\end{abstract}

Index Terms-Memristors, neuromorphic system, crossbar, pattern training and recognition.

\section{INTRODUCTION}

$\mathbf{N}$ euromorphic computation based on memristor is an advanced approach for information processing system [1], and it offers both high density and high connectivity [2]. It is successfully inspired by the human brain which is capable of solving many problems in the areas of data storage [3], pattern-recognition [4], image processing [5] and optimization problem [6, 7].

In [8], multilevel metal oxide memristor is used as synapse in neuromorphic computation. In [9], Park S, Kim H, Choo M, et al present a Resistive Random Access Memory(RRAM) synapse neuromorphic system and confirm its learning capability. Later, a metal-filament based memristive device with stochastic nature is used in neuromorphic system applications in [10]. In [11], a neuromorphic system by using $\mathrm{Al} / \mathrm{Pr}_{0.7} \mathrm{Ca}_{0.3} \mathrm{MnO}_{3}$ (PCMO) memristors is made for character recognition. Moreover, a lot of regular memristor models have been utilized in some specific neuromorphic systems in [12-15]. Recent development in memristor model focuses on memristor with forgetting mechanism [16, 17], which has the feature of "memory loss". However, there are few efforts have been made to develop this device in neuromorphic system.

In [18], Park S, Sheri A, Kim J, et al invent an advanced ReRAM(Resistive Random Access Memory) based synapse neuromorphic speech system and demonstrate its effectiveness. In [19], a hardware system with MCA(Memristor Crossbar

Peijian Zhang, Chuandong Li and Ling Chen are with the Chongqing Key Laboratory of Nonlinear Circuits and Intelligent Information Processing, Department of Electronics and Information Engineering, Southwest University, Chongqing 400715, China

Tingwen Huang is with the Department of Mathematics, Texas A\&M University at Qatar, Doha 23874, Qatar

Yiran Chen is with the Department of Electrical and Computer Engineering,

University of Pittsburgh, PA 15261, USA

*Corresponding author.

E-mail address: cdli@swu.edu.cn(Chuandong Li)

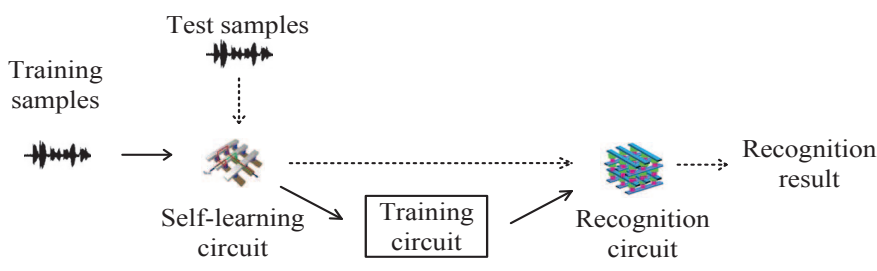

Fig. 1. The process diagram of proposed neuromorphic system for gray handwriting number image recognition, different line patterns represent signal flow of different stages

Array) is presented for visual pattern recognition. This system is tested by binary number images. In [20], a metal-oxide memristor-based integrated neuromorphic system including training and operation is designed.

However most of previous works just focus on either weight training and updating part or recognition part. For example, In [21], a recognition circuit for speech pattern recognition is presented, but its sample learning and weight training is implemented by off-line software without circuit realization. In this study, the whole neuromorphic system is provided in circuit level, both training block and recognition block are designed and operated on line. For exploring the effect of "memory loss" on performance of system learning and recognition, the memristor with forgetting effect is adopted as elementary unit in crossbar array. Simulations not only verify the effectiveness of this system, but also successfully exhibit the relationship between forgetting speed and recognition rate.

The rest of this paper is organized as follows. The proposed system is introduced with detailed explanations of important blocks and learning rule in Section II. As a verification, simulations are presented in Section III, and conclusions are found in Section IV.

\section{SYSTEM DESIGN}

The neuromorphic pattern training and recognition system is shown in Figure 1. Both training samples and test samples are pre-processed. The whole system operation includes three steps: self-learning, weight MCA training and recognition. In following sections, we mainly introduce the self-learning circuit, the training circuit and the recognition circuit.

\section{A. The self-learning circuit}

The schematic of the self-learning crossbar circuit is shown in the top of Figure 2. This MCA has several channel buses and column nanowires (Here is $7 \times 4$ ). Each channel bus consists of some sub-channels (Here is 4), which share the 


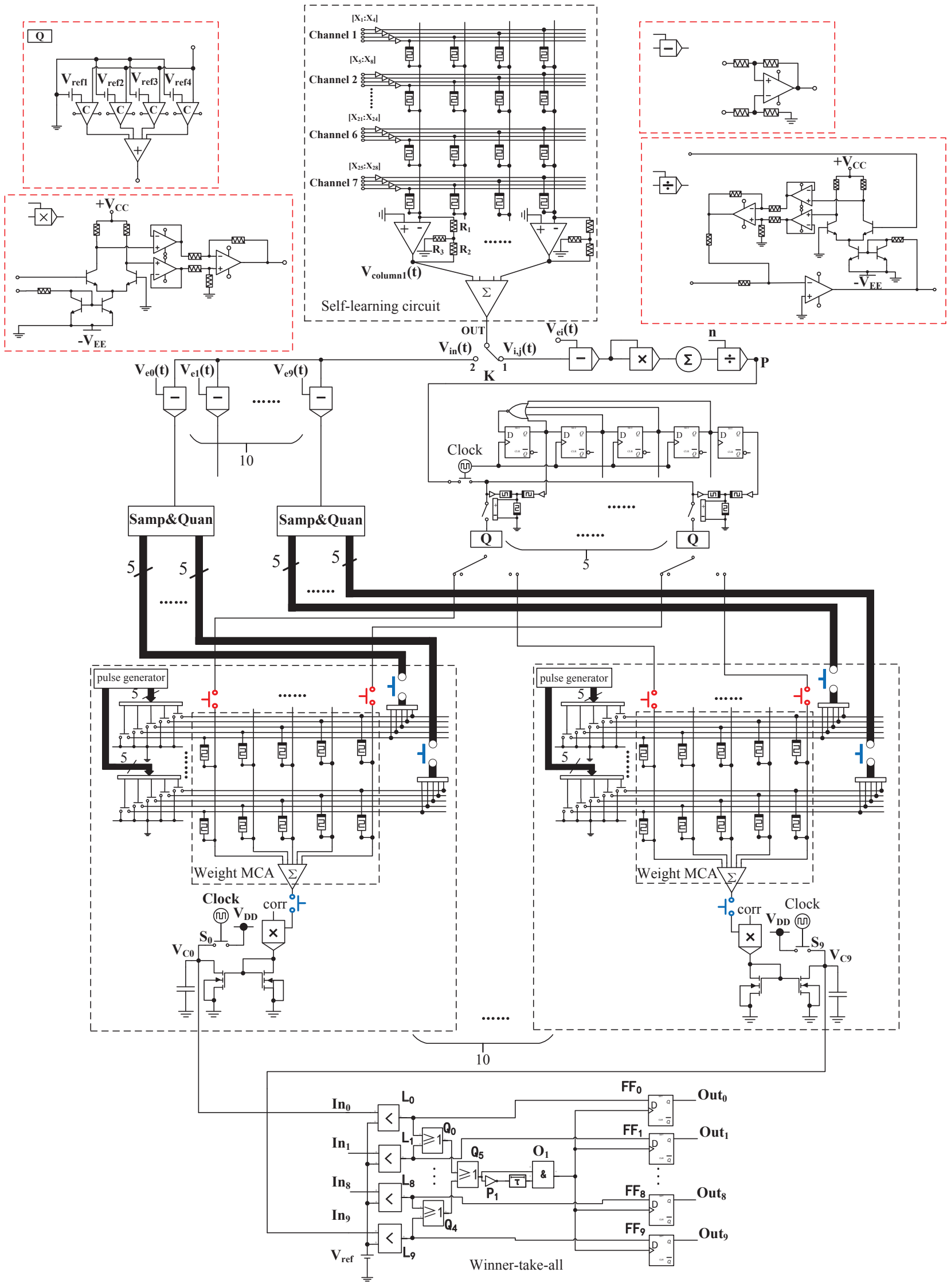

Fig. 2. The schematic of training and recognition circuit 
same column nanowires. At each cross point of channel bus and nanowire, a memristor with forgetting effect is set. After pre-processing, one sample is divided to 28 input signals. If we mark one of those signals as $v_{i}$, the current passing through memristor can be calculated by:

$$
i_{i}(t)=\mathrm{G}\left(v_{i}(t)\right) v_{i}(t)
$$

According to Kirchhoff's law in first column, we can get

$-R_{1}\left(\frac{1}{R_{1}}+\frac{1}{R_{2}}+\frac{1}{R_{3}}\right)\left(i_{4}(t)+i_{8}(t)+\cdots+i_{28}(t)\right)=\frac{v_{\text {column } 1}(t)}{R_{2}}$,

Where $v_{\text {column } 1}(t)$ is output of first operational amplifier. If make $\sum^{\text {column } 1} i_{i}(t)=i_{4}(t)+i_{8}(t)+\cdots+i_{28}(t)$ and $\varepsilon_{1}=$ $R_{1}+R_{2}+\frac{R_{1} R_{2}}{R_{3}}$, the output voltage of first column is given as

$$
v_{\text {column } 1}(t)=-\varepsilon_{1} \sum^{\text {column } 1} i_{i}(t)
$$

Then the output voltage of reverse adder is given by

$$
\begin{aligned}
v_{\text {out }}(t)= & \varepsilon_{1} \sum_{\text {column } 3}^{\text {column } 1} i_{i}(t)+\varepsilon_{2} \sum_{\varepsilon_{3} \sum_{i}^{\text {column }} i_{i}(t)+\varepsilon_{4} \sum^{\text {colum } 4} i_{i}(t) .}
\end{aligned}
$$

As the conductance $G$ is continually changing when current passes through, memristors begin to learn samples. This learning process is carried on by inherent property of memristor, so it is called self-learning process. In this paper, the output of self-learning circuit is called characteristic curve. The learning rule is based on characteristic curve.

\section{B. The learning rule}

The mean variance algorithm is proposed to serve as the learning rule in the weight MCA. Before the weight MCA is trained we obtain the expected signal $v_{e_{i}}$ based on a part of training samples(Here $i$ is serial number of sample category) firstly. Figure 2 shows the whole constitution of the training and recognition circuit. When switch $\mathrm{K}$ is dialed to position 1 while all red switches are closed and all blue switches are open, training mode is started. The training circuit contains some basic analog operational units, detailed circuits are shown in subgraph of Figure 2. These operational circuits are used for computing difference, square and arithmetic mean of signals. If $n$ samples are trained together as a group, the power signal with unit resistance of node $\mathrm{P}$ can be given by

$$
p_{p}(t)=\frac{1}{n} \sum_{j=1}^{n}\left(v_{i, j}(t)-v_{e_{i}}(t)\right)^{2} .
$$

Here $v_{i, j}$ is the characteristic curve of $j$-th training sample of category $i . p_{p}$ is fed into weight-changing circuit. This circuit contains several sequential pulse generators, some memristor-based logic switches [22], a voltage comparison circuit and a crossbar row selector. It is not only a sample-hold circuit but also can separate signals and pass sampled values to different memristor in turn. For clarity, $\hat{p}_{i}^{k}$ is uniformly defined as excitation signal for changing weight MCA. The expression of $\hat{p}_{i}^{k}$ is given as following

$\hat{p}_{i}^{k}(t)=\frac{1}{n} \sum_{j=1}^{n}\left(v_{i, j}(t)-v_{e i}(t)\right)^{2} \cdot[\varepsilon(t-k T)-\varepsilon(t-\tau-k T)]$.

$\varepsilon(t)$ is a unit step function, as a function of continuous variable $t$ :

$$
\varepsilon(t)= \begin{cases}0 & t<0 \\ 1 & t \geq 0\end{cases}
$$

$k$ is the serial number of switch sampling pulse, $\tau$ is the duration of pulse and $T$ is the period. As we can see in Eq. (6), one rectangular pulse of periodic rectangular pulse sequence can be described by $\varepsilon(t-k T)-\varepsilon(t-\tau-k T)$.

In realizing the final conductance of memristor is associated with $\hat{p}_{i}^{k}$, we quantify $\hat{p}_{i}^{k}$ before each memristor is excited by it.

The relationship between conductance and quantized voltage can be written as following

$$
G\left(v_{l}\right) \propto v_{l} .
$$

Here $v_{l}$ have four levels. Quantification is implemented by a comparator circuit. In order to increase the difference, all samples are divided into several groups. Each group can get a power signal $p_{p}$ and modify weight MCA one time. The total conductance of each memristor can be calculated based on the property of non-volatile and Eq. (8).

$$
g_{m}=\sum G\left(v_{l}\right) \propto \sum v_{l}
$$

Finally, the mean variance value is stored in each memristor and serves as a part of features of each category. Training mode is a write procedure. The training circuit processes training sample characteristic signal and modifies weight MCAs by the computed results on-line.

\section{The recognition circuit}

The recognition result is available and visible by using the recognition circuit. When switch $\mathrm{K}$ is dialed to position 2 while all red switches are open and all blue switches are closed, recognition mode is started. The recognition circuit can be divided into three parts: the weight MCAs, the chargedischarge circuit and the winner-take-all circuit. The recognition mode is a read procedure. Recognition circuit classifies test samples by reading category features stored in weight MCAs. $v_{i n}$ is called input voltage which is the characteristic curve of test samples. Difference of $v_{i n}$ and $v_{e i}$ are sampled and inversely proportional quantified, results are served as read voltages.

Each MCA connects with a current mirror charge-discharge circuit. The output current of each MCA is weighted by correlation of test sample and expectation. The biggest current in all MCAs will have the fastest discharge rate.

The winner-take-all circuit is a logical module that can determine which one is the fastest discharged capacitor among all capacitors. Recognition result can be determined by first jump from $\mathrm{Out}_{0}$ to $\mathrm{Out}_{9}$. 


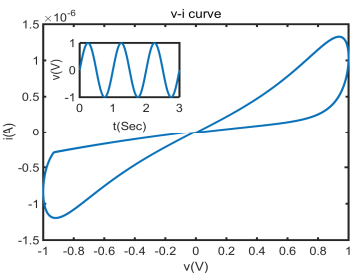

(a) The classical hysteresis loop

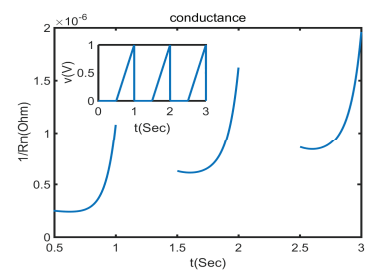

(b) The trend of memristor conductance in consecutive positive voltage

Fig. 3. The simulation of memristor model(the subgraph is input voltage)

\section{Memristor Model}

The memristor we import is one special kind of memristor proposed by Ting Chang et.al [16]. With no excitation, conductance will decrease gradually as time goes by. Its mathematical model is:

$$
\begin{gathered}
i(t)=G(w(t), v(t)) v(t), \\
i(t)=(1-\omega(t)) \alpha[1-\exp (-\beta v(t))]+\gamma \omega(t) \sinh (\delta v(t)), \\
\frac{d \omega(t)}{d t}=\lambda\left(\exp \left(\eta_{1} v(t)\right)-\exp \left(-\eta_{2} v(t)\right)\right) f(\omega(t))-\frac{\omega(t)}{\tau} .
\end{gathered}
$$

The window function $f(\omega(t))$ is:

$$
\begin{aligned}
f(\omega(t)) & =\frac{1}{4}[(\operatorname{sign}(v(t))+1)(\operatorname{sign}(1-\omega(t))+1) \\
& +(\operatorname{sign}(-v(t))+1)(\operatorname{sign}(\omega(t))+1) .
\end{aligned}
$$

Here $G$ is the conductance, $v$ is the voltage worked on memristor, $i$ is the current passing through memristor, $\omega \in[0,1]$ is the inner state of memristor. All the other $\alpha, \beta, \gamma, \eta_{1}, \eta_{2}, \tau$ are positive parameters. In Eq. (12), $-\omega / \tau$ is the term indicates forgetting effect, $\tau$ indicates forgetting speed. The parameter values are set as: $\omega_{0}=0.5, \alpha=0.5 e-6, \beta=0.5, \gamma=4 e-6$, $\delta=2, \lambda=0.0045, \eta_{1}=\eta_{2}=5$ and $\tau=2$ [16]. Its $v-i$ characteristic curve is shown in Figure 3(a). From Eq. (11), the expression of memristor conductance is given:

$g(t)=\frac{1}{v(t)}(1-\omega(t)) \alpha[1-\exp (-\beta v(t))]+\gamma \omega(t) \frac{\sinh (\delta v(t))}{v(t)}$.

As shown in Figure 3(b), there is an approximate exponential relationship between conductance and $t$. The conductance is spontaneous decaying in the period without voltage.

This memristor with forgetting effect is adopted in all MCAs. If $\tau$ is large enough, forgetting effect would be negligibly small. Otherwise, forgetting property would make the system reset naturally as time goes by. This will be further analyzed in discussion part.

\section{SIMULATION AND DISCUSSION}

In this section, training and recognition of hand-writing numbers is regarded as an example for this system. In this paper we used 3000 training samples and 1500 test samples, the self-learning circuit and the training circuit are simulated by SIMULINK and MULTISIM. Schematic diagram of preprocessing is shown in Figure 4. Each gray handwriting

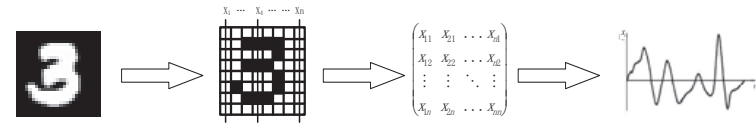

Fig. 4. Image pre-processing
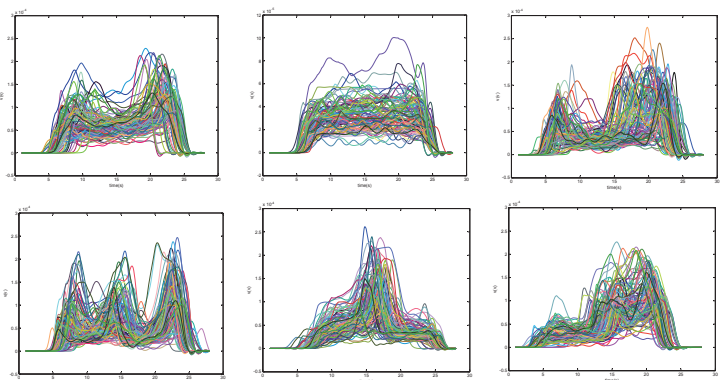

Fig. 5. Some gray images characteristic curves

number image contains a $28 \times 28$ pixel array. These pixel matrix element values are normalized to $[0,1]$, thus all the samples analog voltage signals are under $1.0 \mathrm{~V}$.

Figure 5 shows self-learning output voltage curve of some numbers. Each category sample has typical characteristic curve with different amount and distribution of peaks. These typical characteristic curves are served as the input of the training and recognition circuit. Figure 6 is a distribution diagram of recognition results. Each dot in figure represents a test sample, samples in one category have a same color. The recognition result is indicated by the ordinate. Two numbers have similar characteristic curves are easier to be erroneous recognized. For instance,' 3 ' and '5', '4' and '9'.

The following part is a subsection to discuss the influence of the forgetting mechanism on the proposed system.

\section{A. Forgetting memristor model in system}

Since the self-learning circuit and the recognition circuit are based on memristor device, selection of memristor model is a noteworthy issue in the proposed neuromorphic system. In this system, the memristor model with forgetting effect is chosen as element in all crossbar arrays with infinite value of $\tau$ at the beginning. We modify $\tau$ 's value of memristor crossbar array in

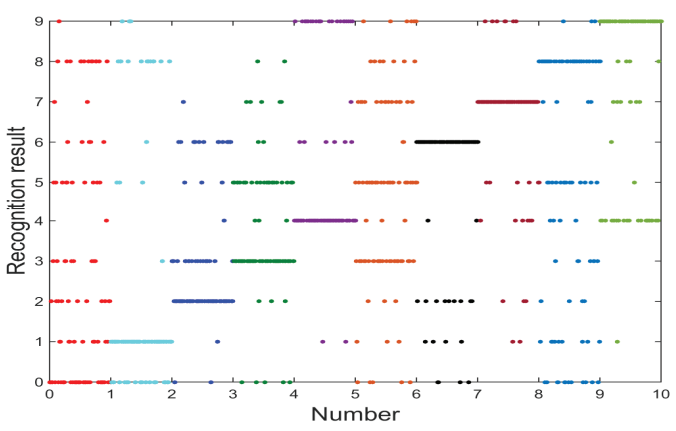

Fig. 6. The distribution diagram of recognition results 


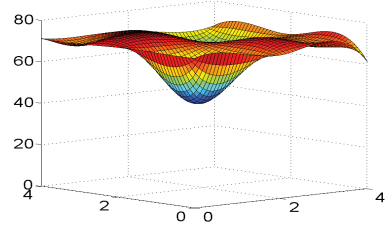

(a) The weight array state at $0 \mathrm{~s}$

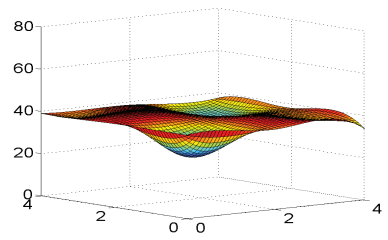

(c) The weight array state at $1.2 \mathrm{~s}$

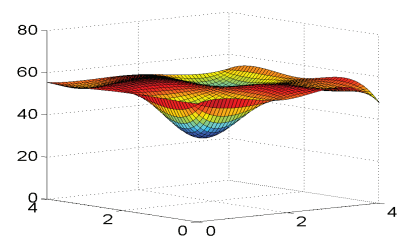

(b) The weight array state at $0.5 \mathrm{~s}$

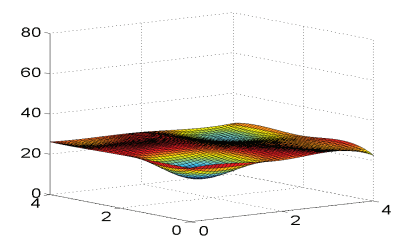

(d) The weight array state at $2 \mathrm{~s}$
Fig. 7. The state changing process of a trained weight MCA vs time

the self-learning circuit and the recognition circuit, but not in memristor logic switch. Forgetting mechanism will decrease recognition result directly, but better match with neuromorphic system. In this part, some simulations and conclusions of relationship between recognition result and $\tau$ 's value are presented.

Due to the forgetting effect in the weight MCAs, which stored the training information, the recognition rate will decrease as time goes by. Figure 7 is a state diagram of a trained weight MCA in 3-D perspective. Characteristics distribution of weight become ambiguous because of the decaying of memristor conductance. Thus, the forgetting process of a weight MCA has been simulated commendably in this figure.

Figure 8 shows some numbers' recognition rate trend versus time with $\tau=2$. The recognition rate shows a downward trend in the whole process. In another aspect, Figure 9 shows the influence of $\tau$ on recognition rate within fixed time. As we can see in this figure, higher recognition rate can be obtained when $\tau$ is increasing. This phenomenon is more obvious when $\tau$ changes from 1.5 to 2.0 .

\section{CONCLUSION}

In this paper, the neuromorphic system with memristor crossbar structure has been presented for pattern training and recognition. In considering of conventional memristor-based crossbar array which has absence of forgetting mechanism of neuromorphic systems, the memristors with forgetting terms are adopted to help research forgetting effect. The proposed learning rule can realize updating of memristor weight by combining with the weight-changing circuit on-line. This proposed neuromorphic system was trained and tested by 4500 image inputs. Simulations indicate that forgetting effect will decrease recognition rate strongly and main influence range is the value of $\tau$ changing from 1.5 to 2.0 .

\section{ACKNOWLEDGMENT}

This work is supported by Natural Science Foundation of China (grant no: 61374078, 61503307), Chongqing Research Program of Basic Research and Frontier Technology (No.

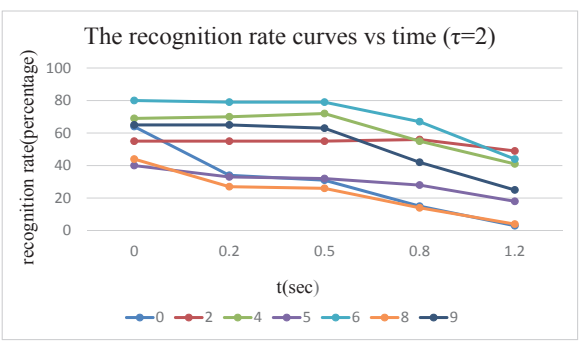

Fig. 8. The recognition rate trend curve of some numbers versus time with $\tau=2$

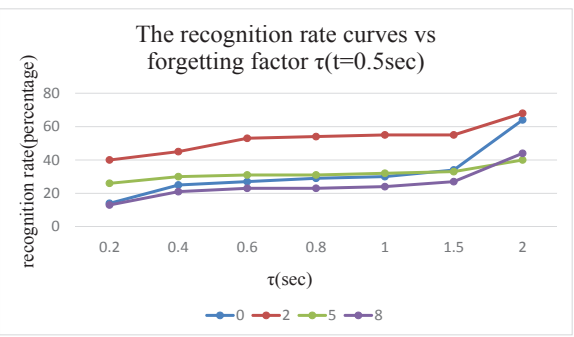

Fig. 9. The recognition rate trend curve of some numbers versus $\tau$ with fixed time $t=0.5 \mathrm{~s}$

cstc2015jcyjBX0052, cstc2016jcyjA0261), NPRP grant NPRP 4-1162-1-181 from the Qatar National Research Fund (a member of Qatar Foundation) and National Science Foundation under grants NSF CNS-1253424 and NFS CCF-1615475. This publication was also made possible by Fundamental Research Funds for the Central Universities (Project No. XDJK2014C118, SWU114007). The statements made herein are solely the responsibility of the authors.

\section{REFERENCES}

[1] Advances in neuromorphic memristor science and applications[M]. Springer Science \& Business Media, 2012.

[2] Jo S H, Chang T, Ebong I, et al. Nanoscale memristor device as synapse in neuromorphic systems[J]. Nano letters, 2010, 10(4): 1297-1301.

[3] Kim K H, Gaba S, Wheeler D, et al. A functional hybrid memristor crossbar-array/CMOS system for data storage and neuromorphic applications[J]. Nano letters, 2011, 12(1): 389-395.

[4] Ebong I E, Mazumder P. CMOS and memristor-based neural network design for position detection[J]. Proceedings of the IEEE, 2012, 100(6): 2050-2060.

[5] Merrikh-Bayat F, Shouraki S B, Merrikh-Bayat F. Memristive fuzzy edge detector[J]. Journal of Real-Time Image Processing, 2014, 9(3): 479-489.

[6] He X, Huang T, Yu J, et al. An inertial projection neural network for solving variational inequalities[J]. 2016.

[7] He X, Li C, Huang T, et al. A recurrent neural network for solving bilevel linear programming problem[J]. IEEE transactions on neural networks and learning systems, 2014, 25(4): 824-830.

[8] Yu S, Wu Y, Jeyasingh R, et al. An electronic synapse device based on metal oxide resistive switching memo- 
ry for neuromorphic computation[J]. Electron Devices, IEEE Transactions on, 2011, 58(8): 2729-2737.

[9] Park S, Kim H, Choo M, et al. RRAM-based synapse for neuromorphic system with pattern recognition function[C]. International Electron Devices Meeting (IEDM). 2012: 10.2 .

[10] Gaba S, Sheridan P, Zhou J, et al. Stochastic memristive devices for computing and neuromorphic applications[J]. Nanoscale, 2013, 5(13): 5872-5878.

[11] Sheri A M, Hwang H, Jeon M, et al. Neuromorphic character recognition system with two PCMO memristors as a Synapse[J]. Industrial Electronics, IEEE Transactions on, 2014, 61(6): 2933-2941.

[12] Liu B, Chen Y, Wysocki B, et al. The circuit realization of a neuromorphic computing system with memristorbased synapse design[C]. Neural Information Processing. Springer Berlin Heidelberg, 2012: 357-365.

[13] Kim H, Sah M P, Yang C, et al. Neural synaptic weighting with a pulse-based memristor circuit[J]. Circuits and Systems I: Regular Papers, IEEE Transactions on, 2012, 59(1): 148-158.

[14] Hu M, Li H, Chen Y, et al. Memristor crossbar-based neuromorphic computing system: A case study[J]. Neural Networks and Learning Systems, IEEE Transactions on, 2014, 25(10): 1864-1878.

[15] Chen L, Li C, Huang T, et al. Memristor crossbar-based unsupervised image learning $[\mathrm{J}]$. Neural Computing and Applications, 2014, 25(2): 393-400.

[16] Chang T, Jo S H, Kim K H, et al. Synaptic behaviors and modeling of a metal oxide memristive device[J]. Applied physics A, 2011, 102(4): 857-863.

[17] Chen L, Li C, Huang T, et al. A synapse memristor model with forgetting effect[J]. Physics Letters A, 2013, 377(45): 3260-3265.

[18] Park S, Sheri A, Kim J, et al. Neuromorphic speech systems using advanced ReRAM-based synapse[J]. IEDM Tech Dig, 2013, 25: 1-25.6.

[19] Chu M, Kim B, Park S, et al. Neuromorphic Hardware System for Visual Pattern Recognition with Memristor Array and CMOS Neuron[J]. Industrial Electronics, IEEE Transactions on, 2015, 62(4): 2410-2419.

[20] Prezioso M, Merrikh-Bayat F, Hoskins B D, et al. Training and operation of an integrated neuromorphic network based on metal-oxide memristors $[\mathrm{J}]$. Nature, 2015, 521(7550): 61-64.

[21] Truong S N, Ham S J, Min K S. Neuromorphic crossbar circuit with nanoscale filamentary-switching binary memristors for speech recognition[J]. Nanoscale research letters, 2014, 9(1): 1-9.

[22] Zhang Y, Shen Y, Wang X, et al. A Novel Design for Memristor-Based Logic Switch and Crossbar Circuits[J]. Circuits and Systems I: Regular Papers, IEEE Transactions on, 2015, 62(5): 1402-1411.

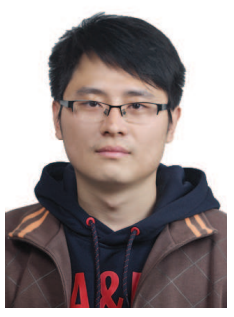

Peijian Zhang received the B.S. degree from Southwest University in College of Electronic and Information Engineering, Chongqing, China, in 2014, where he is currently working toward the M.S. degree. His research interests include neural network, memristor, and image processing etc.

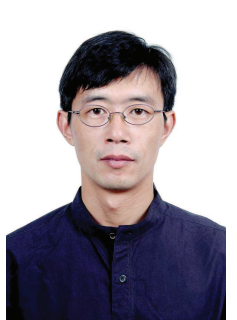

Chuandong Li received his B. S. degree in Applied Mathematics from Sichuan University, Chengdu, China in 1992, and M. S. degree in operational research and control theory and $\mathrm{Ph}$. D degree in Computer Software and Theory from Chongqing University, Chongqing, China, in 2001 and in 2005, respectively. He has been a Professor at the College of Electronic and Information Engineering, Southwest University, Chongqing 400715, China, since 2012, and been the IEEE Senior member since 2010. From November 2006 to November 2008, he serves as a research fellow in the Department of Manufacturing Engineering and Engineering Management, City University of Hong Kong, Hong Kong, China. He has published about more than 100 journal papers. His current research interest covers computational intelligence, neural networks, memristive systems, chaos control and synchronization, and impulsive dynamical systems.

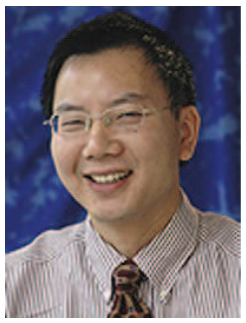

Tingwen Huang obtained his B.S. from Southwest Normal University in 1990, M.S. from Sichuan University in 1993 and Ph.D. from Texas A\&M University in 2002. After he graduated at Texas A\&M University, he has been working in Mathematics Department of Texas A\&M University as Visiting Assistant Professor. In 2003, he started to work at Texas A\&M University at Qatar until now. He now is a professor of Mathematics. His research fields include neural networks, chaos and its applications, etc. He has published about 30 journal papers on neural networks and nonlinear dynamics.

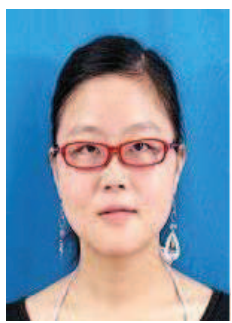

Ling Chen got her B.Sc. from Kunming University of Science and Technology, Kunming, China in 2010, got her Ph.D. from Chongqing University, Chongqing, China. Now she is an assistant professor in Southwest University. Her research interests include neural network, memory devices, memristor, and image processing etc.

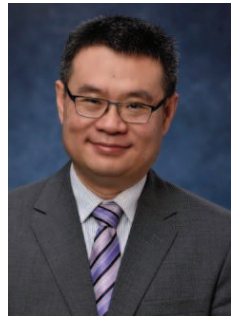

Yiran Chen received B.S and M.S. (both with honor) from Tsinghua University and Ph.D. from Purdue University in 2005. After five years in industry, he joined University of Pittsburgh in 2010 as Assistant Professor and then promoted to Associate Professor in 2014. He is now holding Bicentennial Alumni Faculty Fellow and co-directing Evolutionary Intelligence Lab (www.ei-lab.org) at Electrical and Computer Engineering Department, focusing on the research of nonvolatile memory and storage systems, neuromorphic computing, and mobile applications. 